\title{
Determination of Vibration Parameters in Moderately Damped Systems
}

by BRUCE H. KARNOPP and FRANCIS E. FISHER

Department of Mechanical Engineering, University of Michigan, Ann Arbor, MI 48109, U.S.A.

ABSTRACT: The undamped natural frequency $\omega_{\mathrm{n}}$ and the damping ratio $\zeta$ can be determined from a plot of the free motion of the system. If the damping is light, $\zeta<0.2$, the logarithmic decrement can be used to determine the coefficients. Systems with moderate damping are those in the range $0.2<\zeta<1.2$. A method for determining vibration parameters for systems with moderate damping is presented here. The method is easy to apply and while it is approximate, it is extremely accurate throughout the entire range of moderate damping.

\section{Introduction}

The logarithmic decrement is used to determine the vibration parameters $\omega_{n}$ and $\zeta$ for single degree of freedom systems. This method works very well for systems in which the damping ratio $\zeta$ lies in the range $0<\zeta<0.3$. This range includes the vast majority of important situations.

There are situations of interest in which $\zeta>0.3$. If the system is overdamped, a method exists for determining $\omega_{n}$ and $\zeta(1)$. The method presented here will deal with systems for which $0.2<\zeta<1.2$. This range contains systems which are underdamped : $0.2<\zeta<1.0$; critically damped : $\zeta=1.0$; and overdamped: $1.0<\zeta<1.2$. In each case, the free motion of the system is used to define the vibration parameters. This is worth mentioning here since, from a practical point of view, it is very difficult to distinguish the free motion of systems where $\zeta=0.8,1.0$ and 1.2. While these systems are underdamped, critically damped and overdamped, respectively, their free motions are very similar.

For the sake of discussion, systems in the following ranges are denoted:

$$
\begin{array}{ll}
0.0<\zeta<0.2 & \text { light damping } \\
0.2 \leqslant \zeta \leqslant 1.2 & \text { moderate damping } \\
1.2<\zeta & \text { heavy damping. }
\end{array}
$$

For systems with light damping, the logarithmic decrement method is recommended. For systems with heavy damping, the method of (1) is recommended. Below, a method for systems with moderate damping is developed. 


\section{Systems with Moderate Damping $0.2 \leqslant \zeta \leqslant 1.2$}

The differential equation for the free motion of the system is

$$
\ddot{x}+2 \zeta \omega_{n} \dot{x}+\omega_{n}^{2} x=0 .
$$

Suppose we take $t=0$ to be the time when the solution to (1) has the value $x=0$. Then the solutions to (1) are

$$
\begin{array}{ll}
x=\left(\frac{v_{0}}{\omega_{d}}\right) \mathrm{e}^{-\omega_{n} \zeta t} \sin \left(\omega_{d} t\right) & 0<\zeta<1, \\
x=v_{0} t \mathrm{e}^{-\omega_{n} t} & \zeta=1 \\
x=\left(\frac{v_{0}}{\omega_{d}}\right) \mathrm{e}^{-\omega_{n} \zeta t} \sinh \left(\omega_{d} t\right) & 1<\zeta
\end{array}
$$

where $\omega_{d}=\omega_{n} \sqrt{\left|1-\zeta^{2}\right|}$ and $v_{0}=\dot{x}(0)$. Figure 1 shows typical plots of the solutions (2).

A number of points and measurements will be used in the calculations. These are indicated in Fig. 2. The case shown is of a system with damping ratio $\zeta=0.75$.

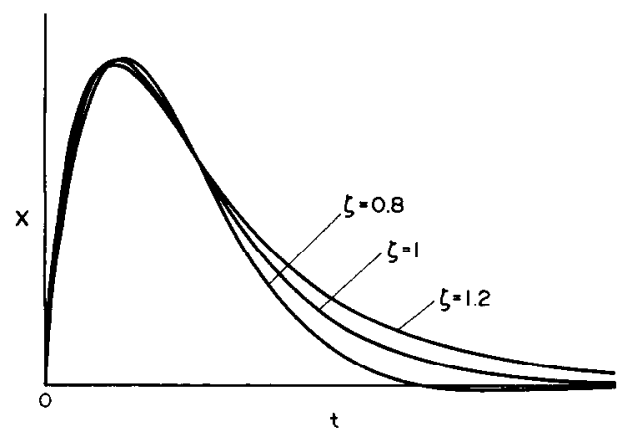

FIG. 1. Typical free motion solutions.

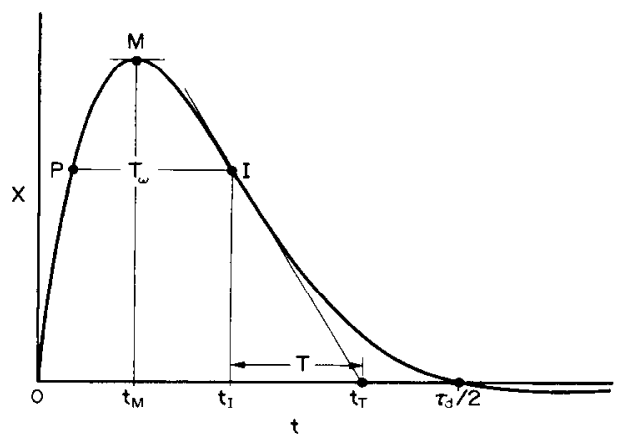

Fig. 2. Parameters used in the computations. 
However, all except one of the parameters will be relevant for all cases of moderate damping.

The following points and quantities will be used in the computation:

$M$-a maximum point of the curve

$x_{M}$ - the displacement at the point $M$

$t_{m}$ - the time of the point $M$

$I$ - the inflection point after $M$

$x_{I}$ the displacement at the point $I$

$t_{i}$ - the time of the point $I$

$t_{T}$ - the time at which a tangent line through $I$ crosses the time axis

$T$ - the time from $t_{I}$ until $t_{T}$

$P$-a point preceding $I$ at the same displacement as $I$

$T_{\omega}$ - the time from $P$ to $I$

$\tau_{d} / 2$ - the half period (for underdamped systems only).

\section{The method}

The method is based upon measurements of $x_{I}$ and $x_{M}$ and the time $T$ (see Figs 2 and 3). Let

$$
R=x_{I} / x_{M}
$$

Then

$$
\zeta=\frac{0.25}{1-R}+0.50 R-0.32
$$

Once $\zeta$ is determined from (4), $\omega_{n}$ is obtained in one of three ways (two ways if the system is critically damped or overdamped).

First :

$$
\omega_{n}=\frac{2 \zeta}{T}
$$

A second method for estimating $\omega_{n}$ results from the measurement of $T_{\omega}$ :

$$
\omega_{n}=\frac{3.0 / T_{\omega}}{1+0.9 \zeta} .
$$

Finally, if the system under consideration is underdamped and the half cycle time $\tau_{d} / 2$ can be determined, the value of $\omega_{n}$ is :

$$
\omega_{n}=\frac{\pi}{\left(\tau_{d} / 2\right) \sqrt{1-\zeta^{2}}} .
$$

\section{A Numerical Example}

Consider the example shown in Fig. 3. Here the (exact) numerical values of $R$, $T_{\omega}, T$ and $\tau_{d}$ are : 


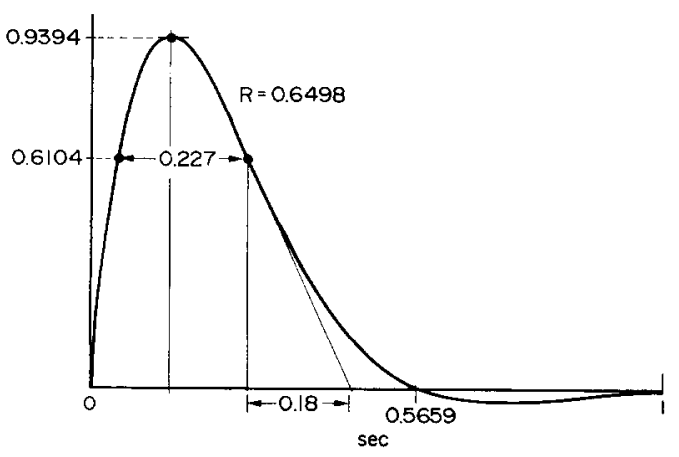

FIG. 3. A numerical example.

$$
\begin{aligned}
R & =(0.6104 / 0.9394)=0.6498 \\
T_{\omega} & =0.2763-0.0493 \mathrm{~s}=0.2270 \mathrm{~s} \\
T & =0.1800 \mathrm{~s} \\
\tau_{d} / 2 & =0.5659 \mathrm{~s} .
\end{aligned}
$$

The exact values are employed here to demonstrate the potential accuracy of the methods proposed. Measured values will not reflect this accuracy, and the results obtained using actual measurements will not have the accuracy of this example. The inaccuracy in that situation is not the fault of the method. From the values in (a), the values of $\zeta$ and $\omega_{n}$ are determined from (4) and (5).

Then

$$
\begin{aligned}
\zeta & =0.719 \\
\omega_{n} & =7.989 \mathrm{rad} / \mathrm{s} \\
\omega_{n} & =8.023 \mathrm{rad} / \mathrm{s} \\
\omega_{n} & =7.988 \mathrm{rad} / \mathrm{s}
\end{aligned}
$$

The exact values in this case are $\zeta=0.72$ and $\omega_{n}=8.0 \mathrm{rad} / \mathrm{s}$.

\section{Proof of the Method}

There are several steps in the proof of (4) and (5):

\section{(1) Location of the Points $\mathrm{M}$ and I}

The maximum point $M$ is easily determined by inspection. Thus the time $t_{M}$ at which $M$ occurs is also determined. It can be shown in all cases that the time at which the curve has an inflection point, $I$, is exactly twice the time for the maximum point: 


$$
t_{l}=2 t_{M} .
$$

To establish (6), the solutions (2) are differentiated and set equal to zero. This gives $t_{M}$. If the second derivatives are set to zero, the times $t_{I}$ result. The times $t_{M}$ and $t_{l}$ for various levels of damping are shown below. In each case, there are simple identities to show that (6) is true. Thus once $M$ is determined, the point $I$ can also be found from a knowledge of $t_{l}$.

\section{(2) Significance of the Point $\mathrm{I}$}

At the point $I$, the acceleration $\ddot{x}$ is zero. Thus the differential equation at $t=t_{t}$ becomes :

$$
0+2 \zeta \omega_{n} \dot{x}_{I}+\omega_{n}^{2} x_{l}=0 .
$$

Thus

$$
\frac{2 \zeta}{\omega_{n}}=-\frac{x_{I}}{\dot{x}_{I}}
$$

From Fig. 2, it is seen that $\dot{x}_{I}=-x_{I} / T$, and thus

$$
\omega_{n}=2 \zeta / T \text {, }
$$

which proves (5a).

In order to use (5a), the value of the damping ratio $\zeta$ must first be determined. The value of $\zeta$ can be obtained from the ratio of $x_{f} / x_{M}$. In order to show that this is possible, it must first be shown that $x_{1} / x_{M}$ is a function of $\zeta$ only. And second, it must be determined if this relation is such that it is effective in determining $\zeta$ from $x_{I} / x_{M}$.

(3) The Ratio $\mathrm{R}=\mathrm{x}_{1} / \mathrm{x}_{\mathrm{M}}$

Suppose $\zeta<1$. By (2) and (6) :

$$
\begin{aligned}
& x_{M}=\left(\frac{v_{0}}{\omega_{d}}\right) \mathrm{e}^{-\omega_{n} \zeta \zeta_{M}} \sin \left(\omega_{d} t_{M}\right) \quad 0<\zeta<1 \\
& x_{I}=\left(\frac{v_{0}}{\omega_{d}}\right) \mathrm{e}^{-\omega_{n} \zeta 2 t_{M}} \sin \left(\omega_{d} 2 t_{M}\right) \quad 0<\zeta<1 .
\end{aligned}
$$

But

$$
\sin (2 \theta)=2 \sin (\theta) \cos (\theta) .
$$

Thus 
TABLE I

\begin{tabular}{|c|c|c|}
\hline Damping ratio $\zeta$ & Time of maximum $t_{M}$ & Time of inflection $t_{I}$ \\
\hline $\begin{array}{c}0 \leqslant \zeta<1 \\
\text { Underdamped }\end{array}$ & $\frac{1}{\omega_{d}} \arctan \left\{\frac{\sqrt{1-\zeta^{2}}}{\zeta}\right\}$ & $\frac{1}{\omega_{d}} \arctan \left\{\begin{array}{c}2 \zeta \sqrt{1-\zeta^{2}} \\
2 \zeta^{2}-1\end{array}\right\}$ \\
\hline $\begin{array}{l}\qquad \zeta=1 \\
\text { Critically damped }\end{array}$ & $\frac{1}{\omega_{n}}$ & $\frac{2}{\omega_{n}}$ \\
\hline $\begin{array}{c}0 \leqslant \zeta<1 \\
\text { Overdamped }\end{array}$ & $\frac{1}{\omega_{d}} \operatorname{arctanh}\left\{\frac{\sqrt{\zeta^{2}-1}}{\zeta}\right\}$ & $\frac{1}{\omega_{d}} \operatorname{arctanh}\left\{\frac{2 \zeta \sqrt{\zeta^{2}-1}}{2 \zeta^{2}-1}\right\}$ \\
\hline
\end{tabular}

$\dagger$ Here the root of the arctangent is laken between 0 and $\pi$.

$$
R=\frac{x_{I}}{x_{M}}=\frac{2 \cos \left(\omega_{d} t_{M}\right)}{\mathrm{e}^{\omega_{n} \zeta_{M}}} .
$$

From Table I,

$$
\tan \left(\omega_{d} t_{M}\right)=\frac{\sqrt{1-\zeta^{2}}}{\zeta}
$$

Thus

$$
\sin \left(\omega_{d} t_{M}\right)=\sqrt{1-\zeta^{2}} \text { and } \cos \left(\omega_{d} t_{M}\right)=\zeta
$$

and

$$
\begin{aligned}
\omega_{d} t_{M} & =\omega_{n} \sqrt{1-\zeta^{2}} t_{M}=\arctan \frac{\sqrt{1-\zeta^{2}}}{\zeta} \\
\omega_{n} \zeta t_{M} & =\frac{\zeta}{\sqrt{1-\zeta^{2}}} \arctan \frac{\sqrt{1-\zeta^{2}}}{\zeta}
\end{aligned}
$$

Equations (7), (8) and (9) show that $R=x_{1} / x_{M}$ is a (complicated) function of $\zeta$. The proofs for the critically damped and overdamped cases is very similar. In principle, a relationship

$$
R=f(\zeta)
$$

has been established. What is desired, however, is the inverse relation $\zeta=F(R)$. And, from the point of view of practical applications, what is desired is a relatively simple expression from which the vibration engineer can determine $\zeta$ quickly and accurately from a measurement of $R$. 


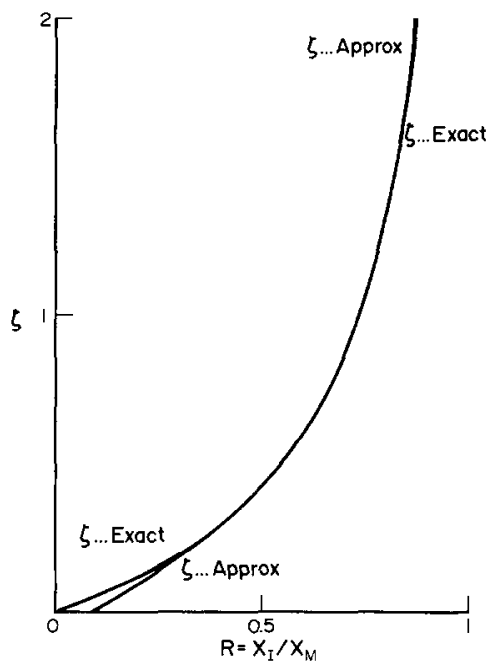

FIG. 4. The $\zeta$ versus $R$ curves.

The relationship $\zeta=F(R)$ is shown as the curve $\zeta \ldots$ Exact in Fig. 4. The following points are worth noting about the exact curve :

(1) For $0 \leqslant \zeta<0.2$, the logarithmic decrement is an effective means of determining $\zeta$. In trying to approximate the exact $\zeta$ curve, the range below 0.2 is unimportant in the current context.

(2) Beyond $\zeta=1.2$, the exact $\zeta$ curve is too steep to be effective in finding $\zeta$. Small errors in $R$ will result in large errors in $\zeta$.

It is reasonable to seek a curve which approximates the exact curve in the range $0.2 \leqslant \zeta<1.2$. Since the exact curve becomes infinite as $R \rightarrow 1$, it is reasonable to try to approximate the curve with a function of the form :

$$
\zeta=\frac{a}{1-R} .
$$

It is reasonable to pick $a$ so the relation is very good at $\zeta=0.7$. This gives $a=0.25$. Temporarily, this function is denoted by $\zeta_{a}$. Subtracting the exact values of $\zeta$ from $\zeta_{a}$, and performing a linear regression on the result gives the following:

$$
\zeta_{a}-\zeta \approx 0.3176-0.4989 R \text {. }
$$

Rounding this off to two decimal places gives:

$$
\zeta \approx \frac{0.25}{1-R}+0.50 R-0.32
$$

This is Eq. (4). The accuracy of (4) is shown in Table II. Between $0.3 \leqslant \zeta \leqslant 1.3$, the error of (4) is $1 \%$ or less. This is certainly acceptable. 
TABLE II

\begin{tabular}{cccr}
\hline Exact & $\zeta$ Fxact & $\zeta(4)$ & \multicolumn{1}{c}{ Frror \% } \\
\hline 0.3025 & 0.2 & 0.1897 & -5.1635 \\
0.4029 & 0.3 & 0.3001 & 0.0468 \\
0.4823 & 0.4 & 0.4041 & 1.0138 \\
0.5986 & 0.6 & 0.6021 & 0.3533 \\
0.6784 & 0.8 & 0.7966 & -0.4296 \\
0.7355 & 1.0 & 0.9929 & -0.7070 \\
0.7785 & 1.2 & 1.1979 & -0.1735 \\
0.7959 & 1.3 & 1.3028 & 0.2184 \\
\hline
\end{tabular}

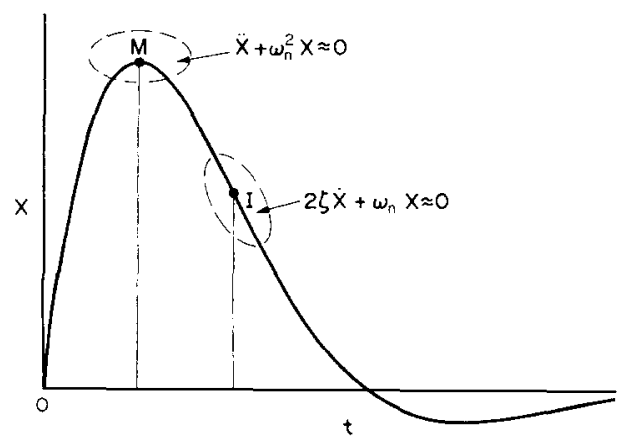

FIG. 5. The points $M$ and $I$.

(4) Proof of (5b)

Consider the plot in Fig. 5. There are two points of interest in the method considered here: $M$ and $I$. At the point $M$, the velocity $\dot{x}=0$. At $I$, the acceleration $\ddot{x}=0$. In the neighborhood of $I$, the solution is that of a first-order system :

$$
0+2 \zeta \omega_{n} \dot{x}+\omega_{n}^{2} x=0 .
$$

On the other hand, in the neighborhood of $M$, the plot resembles that of massspring system :

$$
\ddot{x}+0+\omega_{n}^{2} x=0 .
$$

The solution to $(10)$ is

$$
x=A \sin \left(\omega_{n} t-\phi\right)
$$

Thus the width of the plot about $M$ is, primarily, a function of $\omega_{n}$. If $T_{1,3}$ is the width (time) of the plot from the point $P$ to $I$, see Fig. 2, it is clear that $\left(\omega_{n} T_{w}\right)$ is a function only of $\zeta$. 
TABLE III

\begin{tabular}{cccc}
\hline$\zeta$ & $\omega_{n} T_{\omega}$ Exact & $\omega_{n} T_{\omega}$ Approx. & Error \% \\
\hline 0.0 & $\pi$ & 3.000 & +4.507 \\
0.1 & 2.804 & 2.752 & +1.858 \\
0.2 & 2.553 & 2.542 & +0.416 \\
0.3 & 2.354 & 2.362 & -0.336 \\
0.4 & 2.191 & 2.206 & -0.661 \\
0.5 & 2.054 & 2.069 & -0.709 \\
0.6 & 1.937 & 1.948 & -0.571 \\
0.7 & 1.835 & 1.840 & -0.305 \\
0.8 & 1.745 & 1.744 & +0.052 \\
0.9 & 1.665 & 1.657 & +0.465 \\
1.0 & 1.594 & 1.579 & +0.919 \\
1.1 & 1.529 & 1.508 & +1.404 \\
1.2 & 1.470 & 1.442 & +1.904 \\
1.3 & 1.417 & 1.382 & +2.415 \\
\hline
\end{tabular}

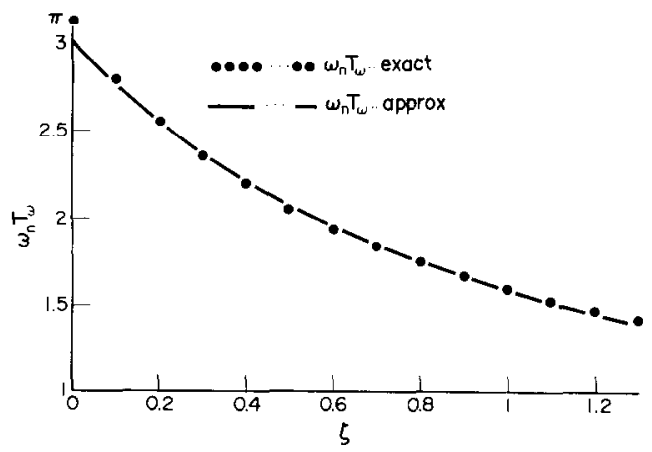

FIG. 6. The $\omega_{n} T_{0}$ versus $\zeta$ curves.

The exact values of $\left(\omega_{n} T_{\omega}\right)$ for various values of $\zeta$ are shown in Table III and are plotted in Fig. 6.

The errors in using (5b) are less than $2 \%$ in the range: $0.1 \leqslant \zeta \leqslant 1.2$. This accuracy is better than one could ordinarily anticipate in making measurements on an experimental plot.

\section{Conclusions}

The determination of $\zeta$ follows from measurement of $R=x_{1} / x_{M}$ and the use of (4). Then $\omega_{n}$ is determined from the time $T$ and (5a) or from the time $T_{\omega}$ and (5b). All computations are easily performed and though the equations are quite simple, the results are very accurate.

It could be argued that the procedure leading to (5b) could be improved by sampling the width of the curve closer to the top at $M$. It is true that looking at 
the curve in the immediate neighborhood of $M$ will give a result independent of $\zeta$. The difficulty with this procedure is that the width of the curve near $M$ is poorly defined. The width of the curve from $P$ to $I$ is, on the other hand, very well defined.

It is also reasonable to question whether the time $\tau_{d} / 2$ might serve as the best way to determine $\omega_{n}$ once $\zeta$ is determined. Again, an examination of Fig. 2 shows that the time $\tau_{d} / 2$ is poorly defined at best. On the other hand, if there are viscous effects present, the time $\tau_{d} / 2$ may be not only poorly defined but irrelevant.

\section{References}

(1) B. H. Karnopp and F. E. Fisher, "On the vibrations of overdamped systems", $J$. Franklin Inst., Vol. 327, pp. 601-609, 1990. 